Canad. Math. Bull. Vol. 46 (4), 2003 pp. 529-537

\title{
Representations of the Twisted Heisenberg-Virasoro Algebra at Level Zero
}

\author{
Yuly Billig
}

Abstract. We describe the structure of the irreducible highest weight modules for the twisted Heisenberg-Virasoro Lie algebra at level zero. We prove that either a Verma module is irreducible or its maximal submodule is cyclic.

\section{Introduction}

In this paper we study the structure of the irreducible representations for the twisted Heisenberg-Virasoro Lie algebra $\mathcal{L}$ at level zero. This Lie algebra is the universal central extension of the Lie algebra of differential operators on a circle of order at most one:

$$
\left\{f(t) \frac{d}{d t}+g(t) \mid f, g \in \mathbb{C}\left[t, t^{-1}\right]\right\} .
$$

The twisted Heisenberg-Virasoro algebra has an infinite-dimensional Heisenberg subalgebra and a Virasoro subalgebra. These subalgebras, however, do not form a semidirect product, but instead, the natural action of the Virasoro subalgebra on the Heisenberg subalgebra is twisted with a 2-cocycle (see (1.1)-(1.3) for the precise definition).

The twisted Heisenberg-Virasoro algebra $\mathcal{L}$ has been studied by Arbarello et al. in $[\mathrm{ACKP}]$, where a connection is established between the second cohomology of certain moduli spaces of curves and the second cohomology of the Lie algebra of differential operators of order at most one. Arbarello et al. also proved that when the central element of the Heisenberg subalgebra acts in a non-zero way, an irreducible highest weight module for $\mathcal{L}$ is isomorphic to the tensor product of an irreducible module for the Virasoro algebra and an irreducible module for the infinitedimensional Heisenberg algebra.

The goal of the present paper is to study the case when the central element of the Heisenberg subalgebra acts trivially (level zero case). It turns out that the picture in the level zero case is quite interesting and very different from the generic case of non-zero level. Our main result (Theorem 1 below) states that either the Verma module itself is irreducible or the irreducible highest weight module is a quotient of two Verma modules. From this we immediately get the characters of the irreducible modules for $\mathcal{L}$ at level zero.

Received by the editors March 8, 2002; revised June 26, 2002.

Research supported by the Natural Sciences and Engineering Research Council of Canada.

AMS subject classification: 17B68, 17B65.

(C)Canadian Mathematical Society 2003. 
Our work is motivated by the representation theory of the toroidal Lie algebras. In order to have a good representation theory for the toroidal Lie algebras one should consider the toroidal algebras together with the Lie algebra of vector fields on a torus. However there are difficulties in representing the toroidal analogue of the Virasoro algebra (see [EM], [FM], [B1], [BBS]). This problem has been resolved in [B2], where the vertex operator representations for the full toroidal algebra are constructed using the twisted Heisenberg-Virasoro algebra. It is precisely the level zero modules that enter in the construction from [B2]. The information about the structure of the irreducible level zero modules obtained in the present paper is required for the computation of the characters of the irreducible representations of the toroidal Lie algebras.

We will denote by $\mathbb{Z}$ the set of integers and by $\mathbb{N}$ the set of natural numbers $\{1,2, \ldots\}$.

Acknowledgements I am grateful to Victor Kac for bringing the paper [ACKP] to my attention.

\section{Twisted Heisenberg-Virasoro Algebra}

We define the twisted Heisenberg-Virasoro algebra $\mathcal{L}$ as a Lie algebra with the basis

$$
\left\{L(n), I(n), C_{L}, C_{L I}, C_{I} \mid n \in \mathbb{Z}\right\}
$$

and the Lie bracket given by

$$
\begin{gathered}
{[L(n), L(m)]=(n-m) L(n+m)+\delta_{n,-m} \frac{n^{3}-n}{12} C_{L},} \\
{[L(n), I(m)]=-m I(n+m)-\delta_{n,-m}\left(n^{2}+n\right) C_{L I},} \\
{[I(n), I(m)]=n \delta_{n,-m} C_{I},} \\
{\left[\mathcal{L}, C_{L}\right]=\left[\mathcal{L}, C_{L I}\right]=\left[\mathcal{L}, C_{I}\right]=0 .}
\end{gathered}
$$

This Lie algebra has an infinite-dimensional Heisenberg subalgebra and a Virasoro subalgebra intertwined with the cocycle (1.2). The twisted Heisenberg-Virasoro algebra $\mathcal{L}$ is the universal central extension of the Lie algebra $\left\{f(t) \frac{d}{d t}+g(t) \mid f, g \in\right.$ $\left.\mathbb{C}\left[t, t^{-1}\right]\right\}$ of differential operators of order at most one. The corresponding projection is given by $L(n) \mapsto-t^{n+1} \frac{d}{d t}, I(n) \mapsto t^{n}$. The center of $\mathcal{L}$ is four-dimensional and is spanned by $\left\{I(0), C_{L}, C_{L I}, C_{I}\right\}$.

We are using the symbol $I$ because we may think of the infinite-dimensional Heisenberg algebra as the affinization of $g l_{1}(\mathbb{C})$. In this interpretation $I$ is the identity matrix.

Introduce a $\mathbb{Z}$ grading on $\mathcal{L}$ by $\operatorname{deg} L(n)=\operatorname{deg} I(n)=n$ and $\operatorname{deg} C_{L}=\operatorname{deg} C_{L I}=$ $\operatorname{deg} C_{I}=0$, and decompose $\mathcal{L}$ with respect to this grading: $\mathcal{L}=\mathcal{L}_{-} \oplus \mathcal{L}_{0} \oplus \mathcal{L}_{+}$.

Irreducible highest weight representations for $\mathcal{L}$ have been studied by Arbarello $e t$ al. in [ACKP], however the case of irreducible representations at level zero, i.e., when $C_{I}$ acts as zero, was not fully investigated in that paper. It turns out that it is precisely 
this type of representations that is needed for the construction of the modules for the toroidal Lie algebras in [B2]. The purpose of the present paper is to describe the structure of the irreducible modules for the twisted Heisenberg-Virasoro algebra at level zero. We are able to give a complete description (Theorem 1 below) of these irreducible modules.

We begin by recalling the standard construction of the Verma modules.

Fix arbitrary complex numbers $h, h_{I}, c_{L}, c_{L I}, c_{I}$. Let $\mathbb{C} 1$ be a 1-dimensional $\mathcal{L}_{0} \oplus \mathcal{L}_{+}$ module defined by $L(0) \mathbf{1}=h \mathbf{1}, I(0) \mathbf{1}=h_{I} 1, C_{L} \mathbf{1}=c_{L} \mathbf{1}, C_{L I} \mathbf{1}=c_{L I} \mathbf{1}, C_{I} \mathbf{1}=$ $c_{I} \mathbf{1}, \mathcal{L}_{+} \mathbf{1}=0$. As usual, the Verma module $M=M\left(h, h_{I}, c_{L}, c_{L I}, c_{I}\right)$ is the induced module

$$
M\left(h, h_{I}, c_{L}, c_{L I}, c_{I}\right)=\operatorname{Ind}_{\mathcal{L}_{0} \oplus \mathcal{L}_{+}}^{\mathcal{L}}(\mathbb{C} \mathbf{1}) \cong U\left(\mathcal{L}_{-}\right) \otimes 1 .
$$

The module $M$ is $\mathbb{Z}$ graded by eigenvalues of the operator $L(0)-h$ Id: $M=$ $\bigoplus_{n=0}^{\infty} M_{n}$ with $M_{n}=\{v \in M \mid L(0) v=(n+h) v\}$.

In order to understand the submodule structure of $M$, we need to study singular vectors in $M$. A non-zero homogeneous vector $v$ in a highest weight $\mathcal{L}$ module is called singular if $\mathcal{L}_{+} v=0$.

Clearly, the highest weight vector $\mathbf{1}$ itself is singular, while every proper homogeneous submodule of a highest weight module contains a singular vector which is not a multiple of the highest weight vector.

The key to the submodule structure of $M$ is the determinant formula derived in [ACKP]. Let us briefly discuss this result.

The Lie algebra $\mathcal{L}$ has an anti-involution $\sigma$ :

$$
\begin{gathered}
\sigma(L(n))=L(-n), \quad \sigma(I(n))=I(-n)-2 \delta_{n, 0} C_{L I}, \\
\sigma\left(C_{L}\right)=C_{L}, \quad \sigma\left(C_{I}\right)=C_{I}, \quad \sigma\left(C_{L I}\right)=-C_{L I} .
\end{gathered}
$$

The Shapovalov form on $M$ is a bilinear form induced by this anti-involution. It has the invariance property

$$
(x u \mid v)=(u \mid \sigma(x) v), \quad x \in \mathcal{L}, \quad u, v \in M,
$$

and normalized by the condition $(\mathbf{1} \mid \mathbf{1})=1$.

Clearly, the distinct graded components of $M$ are orthogonal with respect the Shapovalov form: $\left(M_{n} \mid M_{k}\right)=0$ for $n \neq k$. We consider the restriction of the bilinear form on each component $M_{n}$. If we fix a basis in the space $U_{-n}\left(\mathcal{L}_{-}\right)$then we will get bases in $M_{n}\left(h, h_{I}, c_{L}, c_{L I}, c_{I}\right)$ simultaneously for all $h, h_{I}, c_{L}, c_{L I}, c_{I} \in \mathbb{C}$. Denote by $\operatorname{det}_{n}$ the determinant of the Shapovalov form in this basis. Arbarello et al. established a formula for $\operatorname{det}_{n}$ as a function of $h, h_{I}, c_{L}, c_{L I}, c_{I}$ [ACKP, (6.7)]. In the case when $c_{I}=0$, the determinant formula greatly simplifies. Here we present this reduction. Define the numbers $p_{2}(n)$ by the generating series

$$
\sum_{n=0}^{\infty} p_{2}(n) q^{n}=\prod_{k \geq 1}\left(1-q^{k}\right)^{-2}
$$

and let

$$
\varphi_{r}=\left(h_{I}-(1+r) c_{L I}\right)\left(h_{I}-(1-r) c_{L I}\right), \quad r \in \mathbb{N}
$$


Then

$$
\operatorname{det}_{n}\left(h, h_{I}, c_{L}, c_{L I}\right)=K_{n} \prod_{\substack{1 \leq s \leq r \leq n \\ 1 \leq r s \leq n}} \varphi_{r, s}^{p_{2}(n-r s)},
$$

where

$$
\varphi_{r, s}= \begin{cases}\varphi_{r} \varphi_{s}, & \text { for } r \neq s, \\ \varphi_{r}, & \text { for } r=s,\end{cases}
$$

and $K_{n}$ is a non-zero constant independent of $h, h_{I}, c_{L}, c_{L I}$ (in the notations of [ACKP] take $h_{a}=h_{I}-c_{L I}, c_{3}=i c_{L I}, c_{a}=c_{I}=0$ ).

\section{Structure of the Irreducible $\mathcal{L}$ Modules at Level Zero}

The main result of the paper is the following:

Theorem $1 \quad$ Let $c_{I}=0$ and $c_{L I} \neq 0$.

(a) If $h_{I} / c_{L I} \notin \mathbb{Z}$ or $h_{I} / c_{L I}=1$ then the $\mathcal{L}$ module $M=M\left(h, h_{I}, c_{L}, c_{L I}, 0\right)$ is irreducible.

(b) If $h_{I} / c_{L I} \in \mathbb{Z} \backslash\{1\}$ then $M\left(h, h_{I}, c_{L}, c_{L I}, 0\right)$ possesses a singular vector $v \in M_{p}$, where $p=\left|\frac{h_{I}}{c_{L I}}-1\right|$. The factor-module

$$
L=L\left(h, h_{I}, c_{L}, c_{L I}, 0\right)=M\left(h, h_{I}, c_{L}, c_{L I}, 0\right) / U\left(\mathcal{L}_{-}\right) v
$$

is irreducible and its character is

$$
\operatorname{char} L=\left(1-q^{p}\right) \prod_{j \geq 1}\left(1-q^{j}\right)^{-2} .
$$

Proof Let us give a proof of part (a). Clearly, if there exists a singular vector $v \in$ $M_{n}$ for some $n>0$ then $\operatorname{det}_{n}=0$. Then the determinant formula (1.5) implies that $\varphi_{m}=0$ for some $m \in \mathbb{N}$. It follows from (1.4) that $h_{I} / c_{L I}=1 \pm m$. We conclude that if $h_{I} / c_{L I} \notin \mathbb{Z}$ or $h_{I} / c_{L I}=1$ then the Verma module $M$ does not possess a singular vector other than a vector of the highest weight. Thus the Verma module $M$ is irreducible in this case. This completes the proof of part (a) of Theorem 1.

To prove part (b), we will consider two cases: $1-h_{I} / c_{L I} \in \mathbb{N}$ and $h_{I} / c_{L I}-1 \in \mathbb{N}$. The proof in both cases is essentially the same, so we will treat them in parallel.

Let us first outline the main idea of the proof. The Lie algebra $\mathcal{L}$ has infinitely many Heisenberg subalgebras

$$
\left\langle L(n), I(-n), I(0)-(n+1) C_{L I}\right\rangle_{n \neq 0},
$$

with the Lie bracket

$$
[L(n), I(-n)]=n\left(I(0)-(n+1) C_{L I}\right)
$$


The central element $I(0)-(n+1) C_{L I}$ acts on $M=M\left(h, h_{I}, c_{L}, c_{L I}, 0\right)$ in a non-zero way precisely when $h_{I} / c_{L I} \neq 1+n$. Thus in our case among the Heisenberg subalgebras (2.1), there will be one with a degenerate action on $M$, and the rest will act non-degenerately. We will exhibit a relation between the action of these Heisenberg subalgebras and certain formal operations of taking partial derivatives in $M$ (see Lemma 3 below). The rest of the argument is reminiscent of the classical proof of irreducibility of a polynomial algebra as a module over a Heisenberg Lie algebra.

We will organize the proof of part (b) in a sequence of several lemmas. For the rest of the paper we fix $p=1-h_{I} / c_{L I} \in \mathbb{N}\left(\right.$ resp. $\left.p=h_{I} / c_{L I}-1 \in \mathbb{N}\right)$.

From (1.4) we get that $\varphi_{r}=-c_{L I}^{2}(r-p)(r+p)$. Thus $\varphi_{p}=0$, while $\varphi_{r} \neq 0$ for $r \neq$ $p$, and it follows from the determinant formula (1.5) that $\operatorname{det}_{p}=0$, while $\operatorname{det}_{p-1} \neq 0$. This implies the existence of a singular vector $v \in M_{p}$. Our goal is to show that the submodule $V$ generated by this singular vector is the maximal submodule in $M$. To prove this, we need to study the properties of the singular vector $v$ and of the submodule $V$.

Consider the following Poincaré-Birkhoff-Witt basis in $M=U\left(\mathcal{L}_{-}\right) \mathbf{1}$ :

$$
\left\{I\left(-m_{1}\right) \cdots I\left(-m_{k}\right) L\left(-n_{1}\right) \cdots L\left(-n_{s}\right) \mathbf{1}\right\}
$$

where $m_{1} \geq \cdots \geq m_{k}>0, n_{1} \geq \cdots \geq n_{s}>0$.

Note that the subalgebra $\mathcal{L}_{-}$has one more $\mathbb{Z}$ grading by $I$-degree:

$$
\mathcal{L}_{-}=\left(\mathcal{L}_{-}\right)_{0}^{I} \oplus\left(\mathcal{L}_{-}\right)_{1}^{I},
$$

where $I$-degree of $L(-n)$ is 0 , and $I$-degree of $I(-n)$ is 1 . We place a superscript $I$ in the notation of the graded component in order to distinguish the grading by $I$-degree from the standard grading by the ordinary degree. This new grading on $\mathcal{L}_{-}$induces a $\mathbb{Z}$ grading on $U\left(\mathcal{L}_{-}\right)$and also on the Verma module $M=U\left(\mathcal{L}_{-}\right) \mathbf{1}$ :

$$
M=\bigoplus_{j=0}^{\infty} M_{j}^{I} .
$$

The $I$-degree of a monomial in (2.2) is $k$.

For a non-zero element $w \in M$ we will denote by $\bar{w}$ its lowest non-zero homogeneous component with respect to $I$-degree:

$$
w=\bar{w}+\text { terms of higher } I \text {-degree. }
$$

We define on $M$ the operations of formal partial derivatives $\frac{\partial}{\partial I(-m)}, \frac{\partial}{\partial L(-n)}$. We set

$$
\begin{array}{rlrl}
\frac{\partial I(-j)}{\partial I(-m)} & =\delta_{j m}, & \frac{\partial L(-j)}{\partial I(-m)}=0, & \frac{\partial}{\partial I(-m)} \mathbf{l}=0, \\
\frac{\partial I(-j)}{\partial L(-n)}=0, & \frac{\partial L(-j)}{\partial L(-n)}=\delta_{j n}, & \frac{\partial}{\partial L(-n)} \mathbf{l}=0,
\end{array}
$$

and then define their action on monomials (2.2) by the Leibnitz rule. Finally, we extend these to $M$ by linearity. Clearly, these operations are not canonical and depend on our choice of the basis. 
Lemma 2 Let $w \in M_{k}^{I}$ and let $n>0$. Then

(a) $I(n) w \in M_{k}^{I} \oplus M_{k+1}^{I}$,

(b) $L(n) w \in M_{k-1}^{I} \oplus M_{k}^{I}$.

The proof of this lemma is a simple application of the Poincaré-Birkhoff-Witt argument and is left as an exercise.

We will also need the following subspace in $M$ :

$$
\mathcal{J}=\operatorname{Span}\left\{I\left(-m_{1}\right) \cdots I\left(-m_{k}\right) \mathbf{1} \mid m_{1} \geq \cdots \geq m_{k}>0\right\} .
$$

The next lemma exhibits the relation between the action of the Heisenberg subalgebras (2.1) and the formal partial derivatives.

Lemma 3 Let $w$ be a non-zero vector in M expanded in the basis (2.2), and denote by $k$ the I-degree of its lowest component $\bar{w}$.

(a) Suppose that $\bar{w} \notin \mathcal{J}$. Let $n$ be the smallest integer such that $L(-n)$ occurs as a factor in one of the terms of $\bar{w}$. Then the part of $I(n) w$ of the I-degree $k$ is given by

$$
n\left(h_{I}+(n-1) c_{L I}\right) \frac{\partial \bar{w}}{\partial L(-n)} .
$$

(b) Suppose that $\bar{w} \in \mathcal{J}, \bar{w} \notin \mathbb{C} 1$. Let $m$ be the maximal integer such that $I(-m)$ occurs as a factor in one of the terms of $\bar{w}$. Then the part of $L(m) w$ of the I-degree $k-1$ is given by

$$
m\left(h_{I}-(m+1) c_{L I}\right) \frac{\partial \bar{w}}{\partial I(-m)} .
$$

Proof Let us prove claim (a). By Lemma 2 (a), the part of $I(n) w$ of $I$-degree $k$ comes from $I(n) \bar{w}$. Let

$$
x=I\left(-m_{1}\right) \cdots I\left(-m_{k}\right) L\left(-n_{1}\right) \cdots L\left(-n_{s}\right) \mathbf{1}
$$

be one of the monomials occurring in $\bar{w}$. It is sufficient to establish the claim of the lemma for such a monomial. By our assumption, $n_{1} \geq \cdots \geq n_{s} \geq n$. We have

$$
I(n) x=\sum_{i=1}^{s} I\left(-m_{1}\right) \cdots I\left(-m_{k}\right) L\left(-n_{1}\right) \cdots\left[I(n), L\left(-n_{i}\right)\right] \cdots L\left(-n_{s}\right) \mathbf{1} .
$$

If $n_{i}>n$, we have $\left[I(n), L\left(-n_{i}\right)\right]=n I\left(-n_{i}+n\right)$, and the $I$-degree of the corresponding term will be $k+1$, so such terms will not contribute to the part of $I(n) x$ of $I$-degree $k$.

If $n_{i}=n$, then we have $[I(n), L(-n)]=n\left(I(0)+(n-1) C_{L I}\right)$, and so the contribution of these terms is $n\left(h_{I}+(n-1) c_{L I}\right) \frac{\partial x}{\partial L(-n)}$. Combining these two cases, we obtain claim (a). 
Let us now prove (b). By Lemma 2 (b), the part of $L(m) w$ of $I$-degree $k-1$ comes from $L(m) \bar{w}$. Let

$$
y=I\left(-m_{1}\right) \cdots I\left(-m_{k}\right) \mathbf{1}
$$

be one of the monomials occurring in $\bar{w}$. It is sufficient to establish the claim for such a monomial. By our assumption, $m \geq m_{1} \geq \cdots \geq m_{k}$. We have

$$
L(m) y=\sum_{i=1}^{k} I\left(-m_{1}\right) \cdots\left[L(m), I\left(-m_{i}\right)\right] \cdots I\left(-m_{k}\right) \mathbf{1}
$$

If $m>m_{i}$ then $\left[L(m), I\left(-m_{i}\right)\right]=m_{i} I\left(m-m_{i}\right)$, and the corresponding term vanishes since all $I$ 's commute and $I\left(m-m_{i}\right) \mathbf{1}=0$ when $m-m_{i}>0$.

If $m=m_{i}$, then $[L(m), I(-m)]=m\left(I(0)-(m+1) C_{L I}\right)$, and the contribution of these terms will yield $m\left(h_{I}-(m+1) c_{L I}\right) \frac{\partial y}{\partial I(-m)}$. The proof of the lemma is now complete.

Note that the factor $\left(h_{I}+(n-1) c_{L I}\right)$ in (2.3) vanishes only when $p=1-h_{I} / c_{L I} \in$ $\mathbb{N}$ and $n=p$. The factor $\left(h_{I}-(m+1) c_{L I}\right)$ in (2.4) is zero when $p=h_{I} / c_{L I}-1 \in \mathbb{N}$ and $m=p$. The partial derivatives $\frac{\partial \bar{w}}{\partial L(-n)}$ in (2.3) and $\frac{\partial \bar{w}}{\partial I(-m)}$ in (2.4) are non-zero since by our assumptions $\bar{w}$ involves $L(-n)$ in (a) and $I(-m)$ in (b).

In the following lemma we describe the decomposition of the singular vector $v \in$ $M_{p}$ by $I$-degree.

Lemma 4 The module $M$ possesses a singular vector $v \in M_{p}$ with $\bar{v}=L(-p) \mathbf{1}$ (resp. $\bar{v}=I(-p) \mathbf{1})$.

Proof We have already established the existence of a singular vector $v \in M_{p}$. Denote the $I$-degree of $\bar{v}$ by $k$. Let us reason by contradiction and assume that $\bar{v}$ is not a multiple of $L(-p) \mathbf{1}$ (resp. $I(-p) \mathbf{1})$. If $\bar{v} \notin \mathcal{J}$, we apply Lemma $3(\mathrm{a})$, and find $n \in \mathbb{N}$ such that $I(n) v \neq 0$ (note that in case $p=1-h_{I} / c_{L I} \in \mathbb{N}$ we have $n \neq p$ due to our assumption that $\bar{v}$ is not a multiple of $L(-p) \mathbf{1})$. This contradicts to the fact that $v$ is a singular vector. If $\bar{v} \in \mathcal{J}$ then we also get a contradiction in a similar way. We apply Lemma 3 (b) to find $m \in \mathbb{N}$ such that $L(m) v \neq 0$ (note that in case $p=h_{I} / c_{L I}-1$, we have $m \neq p$ due to our assumption that $\bar{v}$ is not a multiple of $I(-p) \mathbf{1})$. Hence $\bar{v}$ must be a multiple of $L(-p) \mathbf{1}($ resp. $I(-p) \mathbf{1})$ and we rescale $v$ so that $\bar{v}=L(-p) \mathbf{1}$ (resp. $\bar{v}=I(-p) \mathbf{1})$.

Remark In fact it is possible to show that in the case when $p=h_{I} / c_{L I}-1 \in \mathbb{N}$, the singular vector $v$ belongs to $M_{p} \cap \mathcal{J}$.

\section{Example}

(i) If $h_{I} / c_{L I}=0$ then $v=\left(L(-1)+\frac{h}{c_{L I}} I(-1)\right) \mathbf{1}$ is a singular vector of degree 1 in $M\left(h, h_{I}, c_{L}, c_{L I}, 0\right)$.

(ii) If $h_{I} / c_{L I}=2$ then $v=I(-1) 1$ is a singular vector of degree 1 in $M\left(h, h_{I}, c_{L}, c_{L I}, 0\right)$. 
In order to prove Theorem 1 , we need to show that the submodule $V=U\left(\mathcal{L}_{-}\right) v$ generated by the singular vector $v \in M_{p}$ is the maximal submodule in $M$. To achieve this, we will need the following two corollaries to Lemma 4.

Corollary 5 Let $w$ be a non-zero vector in the submodule $V=U\left(\mathcal{L}_{-}\right) v$, written in the basis (2.2). Then there exist terms in $\bar{w}$, containing the factor $L(-p)($ resp. $I(-p))$.

Proof Let $w=u v$, where $u \in U\left(\mathcal{L}_{-}\right)$. Since the universal enveloping algebra $U\left(\mathcal{L}_{-}\right)$ has no zero divisors, we have that $\bar{w}=\bar{u} \bar{v}$. However by Lemma $4, \bar{v}=L(-p) \mathbf{1}$ (resp. $\bar{v}=I(-p) \mathbf{1})$. Thus $\bar{w}=\bar{u} L(-p) \mathbf{1}$ (resp. $\bar{w}=\bar{u} I(-p) \mathbf{1})$. Using the fact that the graded algebra gr $U\left(\mathcal{L}_{-}\right)$associated with the universal enveloping algebra $U\left(\mathcal{L}_{-}\right)$ is isomorphic to a polynomial algebra, we conclude that all the terms in $\bar{w}$ of the maximal length (length of a monomial in (2.2) is $s+k$ ) will contain a factor $L(-p)$ (resp. $I(-p)$ ). Thus we obtain the claim of Corollary 5 .

Corollary 6 The images of the vectors

$$
\left\{I\left(-m_{1}\right) \cdots I\left(-m_{k}\right) L\left(-n_{1}\right) \cdots L\left(-n_{s}\right) \mathbf{1}\right\}
$$

where $m_{1} \geq \cdots \geq m_{k}>0, n_{1} \geq \cdots \geq n_{s}>0, n_{i} \neq p$ (resp. $\left.m_{i} \neq p\right)$, form the basis of the factor module $M / V$.

Proof By Corollary 6, the vectors (2.5) are linearly independent modulo $V$. The character of the subspace in $M$ spanned by these vectors coincides with the character of the factor module

$$
\operatorname{char} M / V=\left(1-q^{p}\right) \operatorname{char} U\left(\mathcal{L}_{-}\right)
$$

Thus the images of the vectors (2.5) under the projection $M \rightarrow M / V$ form the basis of $M / V$.

The next lemma is equivalent to the claim of part (b) of Theorem 1. Our argument here will be quite similar to the one used in Lemma 4 .

Lemma $7 \quad$ Let $w \in M$. If $\mathcal{L}_{+} w \subset V$ then $w \in \mathbb{C} 1 \oplus V$.

Proof Without the loss of generality we may assume that $w$ is homogeneous. Also the statement of the lemma will not change if we add to $w$ a vector from $V$. Applying Corollary 6, we may thus assume that $w$ is a linear combination of vectors (2.5). We need to show that $w \in \mathbb{C} \mathbf{1}$.

If $\bar{w} \notin \mathcal{J}$, then by Lemma 3 (a) there exists $n \in \mathbb{N}$ such that the part of $I(n) w$ of the lowest $I$-degree is non-zero and belongs to the span of (2.5), because the subspace spanned by vectors $(2.5)$ is closed under the operations of taking partial derivatives. However, by the assumption of the lemma, $I(n) w \in V$, which gives us a contradiction to Corollary 5 .

If $\bar{w} \in \mathcal{J}, \bar{w} \notin \mathbb{C} \mathbf{1}$, then by Lemma 3 (b) there exists $m \in \mathbb{N}$ such that the part of the lowest $I$-degree of $L(m) w$ is non-zero and belongs to the span of (2.5). This again 
contradicts to Corollary 5 since $L(m) w \in V$. This leaves us with the only possibility $\bar{w} \in \mathbb{C} \mathbf{1}$ and hence $w \in \mathbb{C} 1$. The lemma is proved.

Finally, to complete the proof of Theorem 1 (b), we note that by Lemma 7 the only singular vectors in $L=M / V$ are multiples of the highest weight vector. Thus $L$ is irreducible.

The formula for the character follows from the obvious equalities

$$
\operatorname{char} L=\left(1-q^{p}\right) \operatorname{char} U\left(\mathcal{L}_{-}\right)
$$

and

$$
\operatorname{char} U\left(\mathcal{L}_{-}\right)=\prod_{j \geq 1}\left(1-q^{j}\right)^{-2}
$$

\section{References}

[ACKP] E. Arbarello, C. De Concini, V. G. Kac and C. Procesi, Moduli spaces of curves and representation theory. Comm. Math. Phys. (1) 117(1988), 1-36.

[BBS] S. Berman, Y. Billig and J. Szmigielski, Vertex operator algebras and the representation theory of toroidal algebras. In: Recent Developments in Infinite-Dimensional Lie Algebras and Conformal Field Theory, Contemp. Math. 297(2002), 1-26.

[B1] Y. Billig, Principal vertex operator representations for toroidal Lie algebras. J. Math. Phys. (7) 39(1998), 3844-3864.

[B2] — Energy-momentum tensor for the toroidal Lie algebras. Preprint, math.RT/0201313.

[EM] S. Eswara Rao and R. V. Moody, Vertex representations for n-toroidal Lie algebras and a generalization of the Virasoro algebra. Comm. Math. Phys. (2) 159(1994), 239-264.

[FM] M. Fabbri and R. V. Moody, Irreducible representations of Virasoro-toroidal Lie algebras. Comm. Math. Phys. (1) 159(1994), 1-13.

School of Mathematics and Statistics

Carleton University

1125 Colonel By Drive

Ottawa, Ontario

K1S 5B6

email: billig@math.carleton.ca 